\title{
Do falling planets cause stellar spin-up?
}

\author{
D. J. A. Brown ${ }^{1}$, A. Collier Cameron ${ }^{1}$, C. Hall ${ }^{1}$ and L. Hebb \\ ${ }^{1}$ SUPA, School of Physics and Astronomy, University of St Andrews, North Haugh, \\ St Andrews, Fife KY16 9SS, UK. \\ email: djab@st-andrews.ac.uk
}

\begin{abstract}
We investigate the tidal interactions between hot Jupiter extra-solar planets and their host stars in an effort to characterise the effects of such interactions on stellar rotation. We study the WASP-18 and WASP-19 systems, showing that in both cases tidal interactions cause the eventual spiral in of the the planet towards the Roche limit. We find that for both systems this process will cause significant spin up of the host star, independent of the precise value for the tidal quality factors. By fitting tidal evolution models to observed parameters, we are able to determine that WASP-19 $\mathrm{b}$ is currently spiralling in, and that it has a very short remaining lifetime $\sim 3 \mathrm{Myr}$.
\end{abstract}

Keywords. stars: rotation, stars: activity, planetary systems

\section{Introduction}

As the number of known extra-solar planetary systems rises, focus is increasingly turning to characterisation of the physical, orbital and spectral properties of both the planets and their host stars. The varied interactions between stars and hot Jupiter planets are also coming under scrutiny in an attempt to improve our understanding of the formation and evolution of planetary systems.

Tidal interactions in the context of binary stars have been extensively studied (e.g. Hut 1980), but interest in their effect on planetary systems has intensified only recently. Previous studies have tended to focus on changes in the orbital parameters (e.g. Mardling \& Lin 2002; Jackson et al. 2008; Barker \& Ogilvie 2009; Jackson et al. 2009) whilst neglecting the rotation of the two bodies, which were shown to play an important role by Dobbs-Dixon et al. (2004). We present work investigating the effects of tidal interactions on the rotation of hot Jupiter host stars, and discuss the applicability of the gyrochronology dating method to hot Jupiter systems.

\section{Gyrochronology}

It is well documented that stars spin down during their main sequence lifetime through the action of magnetic braking. The standard form of this spin down, $P \propto 1 / \sqrt{t}$ (Skumanich 1972), can be derived from $\dot{\Omega} \propto \Omega^{3}$, the simple stellar wind model of Weber \& Davis (1967). This implies that the stellar rotation period can be used as a proxy for stellar age in the absence of any external influence on the stellar rotation.

Gyrochronology, a dating method that takes advantage of this, was developed by Barnes (2007) who showed that there exists a tight, universal relationship between stellar rotation period, age and colour for slowly rotating stars in open clusters. That work has since been used as the basis for studies of a range of stellar clusters (e.g. Collier Cameron et al. 2009; Meibom et al. 2009), all of which have been shown to follow similar relationships. The existence of such a relation for open clusters is not in doubt, 
but the applicability of the gyrochronology model to exoplanet host stars has not been examined in great detail.

\section{Tidal model}

We make use of the tidal equations of Dobbs-Dixon et al. (2004), adding the stellar wind model of Weber \& Davis (1967) to provide a source magnetic braking. We integrate the equations forward in time from a set of initial conditions, ending the integration when the host star reaches its estimated main sequence lifetime or when the planet reaches the Roche limit. We evaluate the integration through a $\chi^{2}$ test of the semi-major axis, eccentricity and stellar spin rate, with the addition of a Gaussian prior on the estimated stellar age to give a test statistic $C$. We take the best fit point in the integration to be the step at which $C$ is a minimum, and take the system age to be the time at that step.

For simplicity we assume that the planet is initially tidally locked, but allow the system to evolve away from this state. We calculate the initial stellar rotation rate by scaling the period-colour relation for the Coma-Berenices cluster (Collier Cameron et al. 2009) to the initial time, which is governed by the observed stellar rotation period and estimated age. $t_{0}$ must be sufficiently early for all possible evolutionary scenarios to be explorable, but late enough that the demonstrable timescale for convergence of the period-colour relation is accounted for.

Our tidal evolution model is built into both a grid search scheme and a Markov Chain Monte Carlo (MCMC) algorithm, to explore a clearly defined, four-dimensional parameter space in $\log \left(Q_{s}^{\prime}\right), \log \left(Q_{p}^{\prime}\right), a_{0}$ and $e_{0}$. The upper limit on $e_{0}$ was defined to avoid the problems with high eccentricity systems discussed by Leconte et al. (2010). We assume monotonic shrinking of the planetary orbit with time, and do not account for stellar obliquity, limiting our investigation to systems that are well-aligned. The best solution returned by the grid search is taken to be that which returns the absolute minimum value of the $C$ test statistic, whilst for the MCMC algorithm the best set of initial parameters are taken to be the median values of the respective individual posterior probability distributions.

For further details of our model we refer the reader to Brown et al. (2011). Here we present the key results from our investigation into the histories of the WASP-18 (Hellier et al. 2009) and WASP-19 (Hebb et al. 2010) planetary systems. These systems were selected owing to pre-existing discrepancies between the age estimates obtained from gyrochronology and stellar model fitting.

\section{WASP-19}

The initial time was set to $0.6 \mathrm{Gyr}$, approximately the age of the Hyades cluster, to limit computation time and ensure that the period-colour relation of Barnes (2007) had converged. The primary region of interest suggested by the grid search was found to contain unphysical solutions caused by the combination of tidal quality factors in that section of our parameter space. We therefore restricted the $\log \left(Q_{s}^{\prime}\right)$ parameter space available to the MCMC algorithm to the secondary region of interest found by the grid search.

Integrating the resulting median MCMC solution indicates that WASP-19 A has an age close to $3 \mathrm{Gyr}$, and that the planet has an extremely short remaining lifetime, of the order of $2 \mathrm{Myr}$, until it reaches the Roche limit. Figure 4 shows that the planet is in the process of its final spiral in, and that the star has been spun up dramatically in the recent past. We investigated a range of tidal quality factors for the same initial eccentricity and 

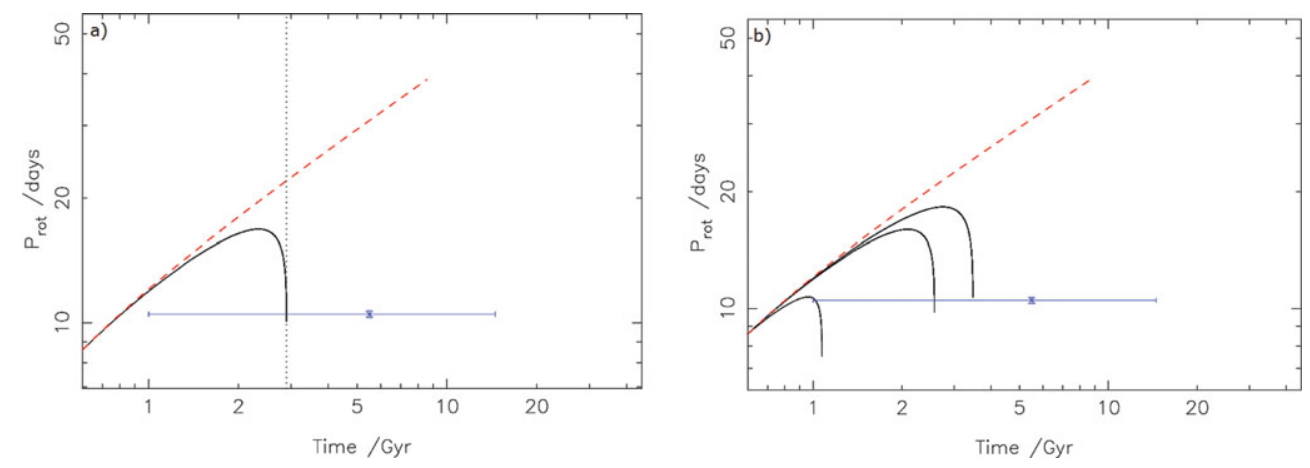

Figure 1. a): Stellar rotation period as a function of time for the median solution obtained by the MCMC algorithm (black, solid line) for the WASP-19 exoplanetary system. Also plotted is the evolution expected from magnetic braking only (red, dashed line). Note the proximity of the observed data (blue datum and error bars) to the end of the evolutionary track, implying a very short remaining lifetime for WASP-19 b. The dotted line denotes the best fit system age derived from the evolutionary track. b): Stellar rotation period as a function of time for a range of $\log \left(Q_{s}^{\prime}\right)$ values consistent with the solution from the MCMC routine. All tracks have the same values of $a_{0}, e_{0}$ and $\log \left(Q_{p}^{\prime}\right)$. In all cases a short remaining lifetime for the planet is implied, and the system has an age that is inconsistent with the estimate obtained using gyrochronology.

semi-major axis, finding a very short $(<3$ Myr) remaining lifetime for WASP-19 b in all cases that were consistent with the observed system parameters. The same evolutionary histories all exhibited a significant, very rapid spin up of WASP-19 A's rotation as the hot Jupiter spiraled in to the Roche limit (figure 4).

The previous lower age limit of 1 Gyr derives from stellar model fitting, and from comparison of the space velocity of WASP-18 A to a simulated population of stars with similar properties (Hebb et al. 2010). This robust limit forces all of the evolutionary tracks to fit the observed period at ages that are entirely inconsistent with the gyrochronology estimate, indicating that stellar spin up must have taken place.

\section{WASP-18}

For the WASP-18 system we set $t_{0}=0.15 \mathrm{Gyr}$, approximately the age of the M35 cluster, to permit the full range of possible solutions to be explored. Integrating the median solution returned by the MCMC algorithm (figure 5) implies that WASP-18 is in a similar situation to WASP-19; a planet on the verge of beginning its final inspiral to the star, a short remaining lifetime for the planet, and a stellar age inconsistent with gyrochronology. This result is somewhat misleading however, as the uncertainty on the age estimate derived from stellar model fitting is such that the star might still be in the magnetic braking only regime of its rotational evolution, and thus gyrochronology might still be applicable.

Figure 5 shows the evolution of the stellar rotation period for a range of $\log \left(Q_{s}^{\prime}\right)$ values consistent with the MCMC result, and with the same $a_{0}, e_{0}$ and $\log \left(Q_{p}^{\prime}\right)$. These tracks imply a large range of potential ages for the star, some of which suggest that tidal effects have yet to affect its rotation and that it therefore has a young age consistent with gyrochronology. The evolutionary status of WASP-18 b is therefore considerably less certain than that of WASP-19 b, but it is important to note that all of the tracks show considerable spin-up of the host star towards the end of the planet's lifetime. 

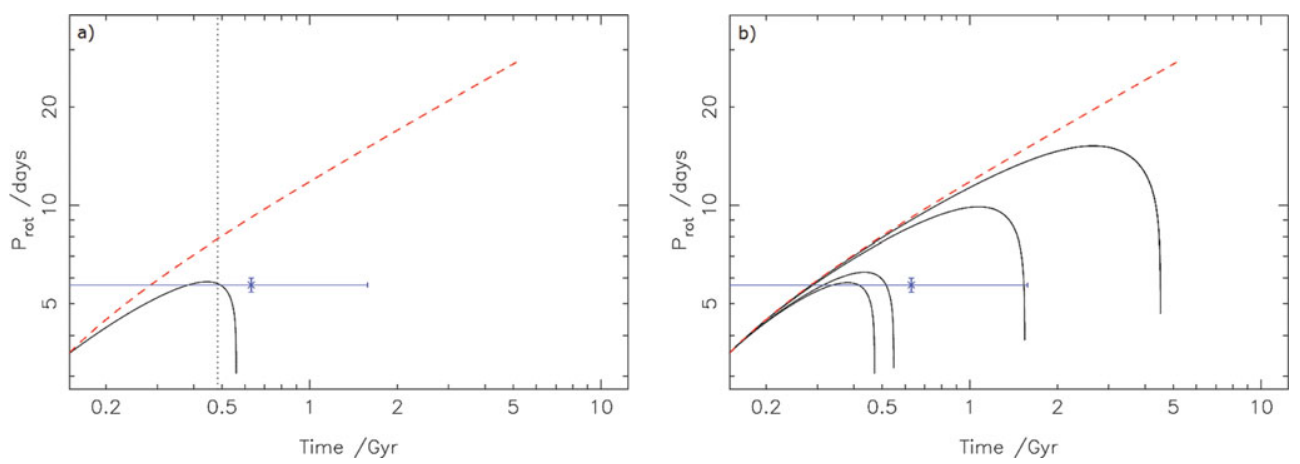

Figure 2. a): Stellar rotation as a function of time for the WASP-18 system. Legend as for figure 1. The track implies that the hot Jupiter is on the verge of spiralling-in, and that the star will be significantly spun up whilst this occurs. b): As figure 4. For this system the range of evolutionary histories suggest a large range of possible ages, some of which are consistent with the gyrochronological estimate. In all cases stellar spin up is implied at the end of the planet's lifetime.

\section{Conclusions}

From our study of the WASP-18 and WASP-19 exoplanetary systems we are able to conclude that tidal interactions between hot Jupiters and their host stars can have a strong effect on rotation of the host star, with the final infall of the planet towards the Roche limit causing notable spin up. This will manifest itself in stellar age estimates, with stars appearing younger as a result of their rotation than implied by their other properties. This signature could potentially be used to detect planet hosting stars.

We also conclude that although gyrochronology is a useful tool, due care should be taken with its application to hot Jupiter systems, as tidal spin up will cause the star to appear much younger than its true age if the technique is applied blindly. This is aptly illustrated by the WASP-19 system, which is certainly older than implied by its rotation. Conversely, the WASP-18 system shows that gyrochronology can give usful indications as to a hot Jupiter host's age, particularly where existing methods appear to give results consistent with the technique.

\section{References}

Barker, A. J. \& Ogilvie, G. I. 2009, MNRAS, 395, 2268

Barnes, S. A. 2007, ApJ, 669, 1167

Brown, D. J. A, Collier Cameron, A., Hall, C., Hebb, L., \& Smalley, B. 2011, MNRAS, 415, 605

Collier Cameron, A., Davidson, V. A., Hebb, L., Skinner, G., et al. 2009, MNRAS, 400, 451

Dobbs-Dixon I., Lin D. N. C. \& Mardling R. A. 2004, ApJ, 610, 464

Hebb, L., Collier-Cameron, A., Triaud, A. H. M. J., Lister, T. A., et al. 2010, ApJ, 708, 224

Hellier, C., Anderson, D. R., Cameron, A. C., Gillon, M., et al. 2009, Nature, 460, 1098

Hut, P. 1980, A\&A, 92, 167

Jackson, B., Greenberg, R., \& Barnes, R. 2008, ApJ, 678, 1396

Jackson, B., Barnes, R., \& Greenberg, R. 2009, ApJ, 698, 1357

Leconte, J., Chabrier, G., Baraffe, I., \& Levrard, B. 2010, A\&A, 516, A64

Mardling, R. A. \& Lin, D. N. C. 2002, ApJ, 573, 829

Meibom, S., Mathieu, R. D., \& Stassun, K. G. 2009, ApJ, 695, 679

Skumanich, A. 1972, ApJ, 171, 565

Sozzetti, A., Torres, G., Charbonneau, D., Latham, et al. 2007, ApJ, 664, 1190

Weber, E. J. \& Davis Jr., L. 1967, ApJ, 148, 217 